\title{
Smart Walking Stick for Visually Impaired People Using Ultrasonic Sensors and Arduino
}

\author{
Dada Emmanuel Gbenga ${ }^{\# 1}$, Arhyel Ibrahim Shani ${ }^{\# 2}$, Adebimpe Lateef Adekunle ${ }^{* 3}$ \\ \# Department of Computer Engineering, "Department of Computer Science, \\ ${ }^{1,2}$ University of Maiduguri, Maiduguri, Borno State, Nigeria \\ ${ }^{3}$ Emmanuel Alayande College of Education, Oyo, Oyo State, Nigeria \\ 1 gbengadada@unimaid.edu.ng \\ 2xarhyelmails@gmail.com \\ 3radebimpela@yahoo.com
}

\begin{abstract}
This paper presents the smart walking stick based on ultrasonic sensors and Arduino for visually impaired people. There are approximately 37 million people across the globe who are blind according to the World Health Organization. People with visual disabilities are often dependent on external assistance which can be provided by humans, trained dogs, or special electronic devices as support systems for decision making. Thus, we were motivated to develop a smart white cane to overcome these limitations. We accomplished this goal by adding ultrasonic sensors at specific positions to the cane that provided information about the environment to the user by activating the buzzer sound. We proposed low cost and light weight system designed with microcontroller that processes signal and alerts the visually impaired person over any obstacle, water or dark areas through beeping sounds. The system consists of obstacle and moisture detection sensors for receiving, processing and sending signals to the alarm system which finally alerts the user for prompt action. The system was designed, programmed using $C$ language and tested for accuracy and checked by the visually impaired person. Our device can detect obstacles within the distance of about $2 \mathrm{~m}$ from the user.
\end{abstract}

Keyword - Ultrasonic sensor, Arduino ATmega328 Microcontroller, Mobility aid, Visually Impaired Person, Alarm system

\section{INTRODUCTION}

Visually impaired people are the people who finds it difficult to recognize the smallest detail with healthy eyes. Those who have the visual acuteness of 6/60 or the horizontal range of the visual field with both eyes open have less than or equal to 20 degrees. These people are regarded as blind. A survey by WHO (World Health Organization) carried out in 2011 estimates that in the world, about $1 \%$ of the human population is visually impaired (about 70 million people) and amongst them, about 10\% are fully blind (about 7 million people) and $90 \%$ (about 63 million people) with low vision according to [1]. The main problem with blind people is how to navigate their way to wherever they want to go. Such people need assistance from others with good eyesight. As described by WHO, 10\% of the visually impaired have no functional eyesight at all to help them move around without assistance and safely. The figure 1 below is a chart showing the percent of blind people across the globe. This study proposes a new technique for designing a smart stick to help visually impaired people that will provide them navigation. The conventional and archaic navigation aids for persons with visual impairments are the walking cane (also called white cane or stick) and guide dogs which are characterized by a many imperfections. The most critical shortcomings of these aids include: essential skills and training phase, range of motion, and very insignificant information communicated been communicated. Our approach modified this cane with some electronics components and sensors, the electronic aiding devices are designed to solve such issues. The ultrasonic sensors, water sensor, buzzer, and RF transmitter/Receiver are used to record information about the presence of obstacles on the road. Ultrasonic sensor have the capacity to detect any obstacle within the distance range of $2 \mathrm{~cm}-450 \mathrm{~cm}$. Therefore whenever there is an obstacle in this range it will alert the user. Water sensor is used to detect if there is water in path of the user. Most blind guidance systems use ultrasound because of its immunity to the environmental noise. With the rapid advances of modern technology both in hardware and software it has become easier to provide intelligent navigation system to the visually impaired. 


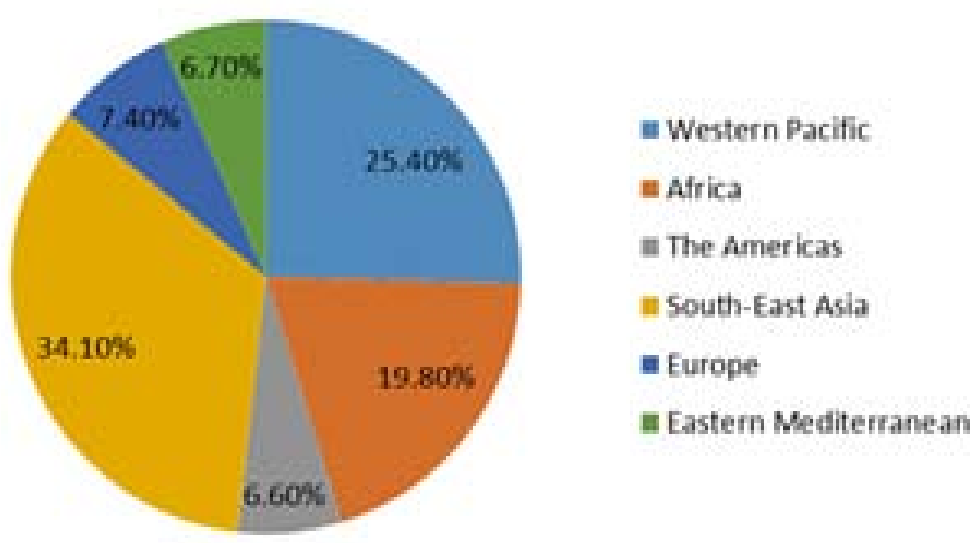

Fig. 1: A Pie Chart Showing Blind People Across the World

Recently, much research effort have been focused on the design of Electronic Travel Aids (ETA) to aid the successful and free navigation of the blind. Also, high-end technological solutions have been introduced recently to help blind persons navigate independently. Another reason why ultrasonic is prevalent is that the technology is reasonably cheap. Moreover, ultrasound emitters and detectors are portable components that can be carried without the need for complex circuit. RF module will help the person to find the stick wherever it is placed. Whenever the user wants to locate it, such a person will press a button on remote control and buzzer will ring, then the person can get the idea of where the stick is placed.

Vision is the most important part of human physiology as $83 \%$ of information human being gets from the environment is via sight. The 2011 statistics by the World Health Organization (WHO) estimates that there are 70 million people in the world living with visual impairment, 7 million of which are blind and 63 million with low vision. The conventional and oldest mobility aids for persons with visual impairments are characterized with many limitations. Some inventions also require a separate power supply or navigator which makes the user carry it in a bag every time they travel outdoor. These bulky designs will definitely make the user to be exhausted. The objectives of this research work include: to design an assistive technology for visually impaired people that can detect obstacles and provides alternative routes for the blind; to alarm the user through vibration to determine the obstacles direction sources; and to help the user find his stick when he cannot remember where is was kept.

Several attempts have been made to design guard or obstacle avoidance devices for the blind using components with limited number of applications. This section will discuss some of these attempts and their shortcomings. For instance; [2] proposed a Smart Walking Stick for Visually Impaired. The proposed method is a simple walking stick equipped with sensors to give information about the environment. GPS technology integrated with pre-programmed locations allows the user to choose the optimal route to be taken. In the system, ultrasonic sensor, pit sensor, water sensor, GPS receiver, level converter, driver, vibrator, voice synthesizer, keypad, speaker or headphone, PIC16F877A microcontroller and battery were used. The source code for the PIC microcontroller was developed with MPLAB software. The proposed system intended to provide low cost and efficient navigation aid for the blind which gives a sense of artificial vision by providing information about the environmental scenario of objects around them whilst providing real-time assistance via GPS. The performance of the prototype developed was evaluated with four obstacle-scenario which are: Concrete wall, Human body, Cardboard box, and Plastic. The proposed solution is a moderate budget navigational aid for the visually impaired. As far as localization is concerned, it will be able to provide accurate details of the location of the blind in case they get lost via the GPS. The developed prototype gave good results in detecting obstacles placed at a distance in front of the user. Obstacles and pit can be determined easily by sensor readings. The cost effectiveness of the proposed solution leads to compromises in performance. One of the drawbacks of their proposed method is that the capability of the prototype is limited as a visually impaired person can travel only to four locations using the stick. Also, the navigation system will need to convey information other than that needed for guidance, and it is not feasible to provide guidance information at high intermittencies. It did not provide the functionality for voice control using speech recognition. Other improvements that could have improved the proposed system include: Increasing the range of the ultrasonic sensor and implementing a technology for determining the speed of approaching obstacles. Synchronization with external memory to increase the number of routes stored. Synchronization with various navigation software applications available on the internet so that new, un-programmed destinations can also be chosen. Integration of a GSM module for safety purposes. 
Reference [3] designed and implemented a Smart Stick for Obstacle Detection and Navigation. Their proposed system utilized infrared, ultrasonic and water sensors. It also used GPS and GSM module. GPS to give positioning and navigation to the stick. GSM module helps to give notifications when the blind person is faced with threats. The system is powered by a rechargeable battery. The hardware implemented on their proposed system consists of the Pair of ultrasonic sensors, Infrared sensor, Water sensor, GPS module, GSM/GPRS module, and Arduino Uno microcontroller board (ATmega328P). The smart stick facilitates the blind person to make calls at times of emergency via the GSM/GPRS module. The GPS module also helps to trace the blind person through the data collected by it. It warns the blind person through beep sound whose intensity increases as the person nears the obstacle which aid him to move aside of the obstacle. Also, when obstacles are detected, it invokes the right speech warning message through a Bluetooth earphone. The use of a rechargeable battery in the system also ensures longer time usage. Their proposed system can also detect obstructions that are hidden such as downward stairs, holes etc. The drawback of this proposed stick is that it can be difficult to keep because it was not designed to be foldable. Modification to the proposed system would be: A Braille input device to give the blind person an uncomplicated method to provide the destination address for navigation. Programmable wheels to steer the stick away from the obstacles and also lead the blind person towards his/her destination. Employing IoT to give the benefits of inter-communication between smart sticks (or mobile, PCs) nearby to utilize the functionality of the other stick when one stick's functionality breaks down. Also, running this integrated set of hardware requires an alternative to the battery. The use of solar panels for instance, will be more advantageous in order to get recharged. Obstacles within the distance of about $3 \mathrm{~m}$ can be detected with the help of these sensors.

Reference [4] proposed a Smart Walking Stick which is an Electronic Approach to Assist Visually Disabled Persons. Their device is a microcontroller based automated hardware that can assist a blind to detect obstacles in front of him/her promptly. The hardware consists of a microcontroller PIC16F690 incorporated with ping sonar sensor, proximity sensor, wet detector, a GH311 Ultrasonic obstacle sensor, a micro pager motor and additional equipment. The simplicity of the proposed design makes it easy to use by any person and at the same time the cost of manufacturing such sticks is kept low. The power consumption of the proposed stick is low and can be operated easily. It is also very cheap compared to the conventional ones. Obstacle and hole can be determined easily by sensor readings. The design has an added vibratory feedback mechanism necessary for creating vibratory signal for multiple disable persons to get precise information from the output. Also, the microcontroller can be code-protected so that its security cannot be overridden except by the user or vendor. Wet, muddy or possibly slippery terrain can be detected by a pair of electrodes. Apart from others blind guidance systems; it has a fingernail controller. This provides mechanical advantage beyond anyone's imagination. Running this integrated set of hardware requires an alternative to the battery. The use of solar panels for instance, will be more advantageous in order to get recharged. The proposed stick is not bendable therefore keeping it might be challenging. This cost effective and light weight device can be designed to take the pattern of a plastic and portable device which can be completely fixed on the familiar white cane or blind stick.

In [5] the Voice Enabled Smart Walking Stick for Visually Impaired persons was proposed. Their proposed system consists of a simple walking stick equipped with ultrasonic sensors to give information about the environment such as object detection, pit sensing and water sensing. GPS technology is integrated with preprogrammed locations to determine the optimal route that the blind should navigate. Also, a voice enabled equipment switching is provided to help the blind person in private domain. The proposed system used two Ultrasonic detectors which are Pit sensor and Water sensor; GPS receiver, GSM module, Voice synthesizer, ATmega328/P microcontroller, relay, speaker and battery. The GSM module and relay are for the purpose of switching on the equipment. It helps in transferring the information about the needed operation to be performed on the equipment and generates the resultant switching action. The central merit of this system is that it helps the blind people in both inside and outside, care-free navigation. The GPS based blind device with user input interface signals the blind person when he/she reaches the destination by voice. The integration of room equipment switching along with this system makes it even more useful, thus making it suitable for both indoor and outdoor environment. The smart stick not only aids in detecting obstructions placed at a distance in front of the user, but also provides real time assistance via GPS. The information regarding barriers is given through voice alerts that eradicate the problem of understanding vibration patterns which was used in previous systems. Their system is a reasonable budget navigational aid for the visually impaired. The cost effectiveness of the proposed solution leads to compromises in performance. Some improvements that could be made are as follows: Increasing the range of the ultrasonic sensor, and implementing a technology to determine the speed of the approaching obstacle. Also, running this integrated set of hardware requires an alternative to the battery. It should also accommodate wide varying grips for flexible handling. Their proposed combination of various working units makes a real-time system that monitors position of the user and provides dual feedback making navigation more safe and secure. 
Reference [6] proposed Smart White Cane which is a Sophisticated and Economic Walking Aid. Their proposed stick is designed to detect obstacles which may help the blind to navigate carefree. Their device is made up of components such as ATMega328PU microcontroller, 4 HC-SR04 Ultrasonic Sensor Modules, Sound IC-APR33a3, Vibration Motor, headphones and battery. Audio feedback. Their method alerts users by pre-recorded sound messages and a haptic feedback in form of vibrations. The stick can detect pits, potholes, downfalls, staircase (up and down), low lying and knee level obstacles and even those above the waist. Their system is a moderate budget navigational aid for the visually impaired. The entire circuitry along with the battery cubicle is hidden within the stick thereby decreasing the risk of damage to the circuit and making the device less bulky. The system provides ON/OFF switch, vibration feedback and the audio jack on the handle itself. The system does not have a global positioning method to find the position of' the user using the GPS and guidance to their destination given to the user by voice navigation. The stick does not have the ability to detect oncoming vehicle, slippery floor, and there is no fire or smoke alarm. The idea behind the design of the stick was to keep it structurally similar i.e. thin, lightweight and easy to handle, yet give an active feedback to the user regarding hazards in his walking path.

In [7] an Intelligent Walking Stick for the Blind was proposed. The proposed navigation device for the visually impaired is focused on providing voice output for obstacle navigation using infrared sensors, RFID technology, and android devices. The device has proximity infrared sensors; RFID tags are installed into public building and also integrated into blind person's walking stick. The device is connected to an android phone through Bluetooth. An android application is designed which gives voice navigation based on RFID tag read and also updates person's location information on the server. Another application is designed for family members to access the blind person's location through the server whenever needed. The components of the system are: AT89C51 microcontroller, Bluetooth HC05, MAX232, ADC 0808 and IR sensors, RFID sensor, Android phone, Server and Android application. The system can be used both indoor and outdoor navigation. Blind person's location can be tracked whenever needed which will ensure additional safety. Their approach have the ability to detect obstacles and alerts the blind person through vibration alert and speech output. The drawback of their approach is that it is not compact. Also, use of active RFID tags will transmit the location information automatically to the PCB unit, when the intelligent stick is in its range. The RFID sensor does not have to read it explicitly. The whole device is designed to be small and used in conjunction with the white cane.

Reference [8] proposed Smart Electronic Stick for Visually Impaired. Their proposed system is a theoretical model and a system concept to provide a smart electronic aid for blind people. The system is intended to provide artificial vision, object detection and emergency messaging facility. Ultrasonic sensors calculate the distance of the obstacles around the blind person to guide the user towards the available path. Output is in the form of beeps which the blind person can hear. GPS and GSM are used to acquire the exact location of the blind person at times of emergency and send the co-ordinates to his/her relatives. The hardware consists of Arduino mega board ATmega2560, ultrasonic sensor, Infrared sensor, GPS, GSM, Keypad and two speakers. The sensors used in the stick proposed are highly accurate and sensitive. They provide exact readings of obstacles and distance to be travelled. The GPS and GSM modules provides the location of the patient and thus help the patient in time of need by sending an emergency messaging. The speaker helps in human-machine interface by sending the signals to the patient about obstacles and route to be travelled. Special lineament like extra secondary IR/laser sensor package, remote monitoring package, weather monitoring package and other hardware can be integrated in the design. Also, running this integrated set of hardware requires an alternative to the battery. Their proposed stick was intended to provide low cost and efficient navigation aid for the blind.

Reference [9] proposed a multidimensional walking aid for visually impaired using ultrasonic sensors network with voice guidance. The proposed method implemented a network of ultrasonic sensors capable of detecting the direction and position of obstacle(s). The performance and functionality are improved by the addition of alert light, and voice guidance signal which is relayed to a miniature headset. Hardware used in the implementation of the system are ISD 2590 voice record/playback chip, PIC16F887 microcontroller, ultrasonic sensors, voltage regulator, and speakers (headset and loudspeaker). The recorded voice alerts the user of the presence and direction of the obstacle(s). The prototype of the visually impaired aid is able to detect obstacles in all directions of the user. The performance of his proposed stick in detecting obstacles is low, that is, 1 meter maximum range of detection. The walking stick cannot determine the distance of the obstacle to the multidimensional. The prototype of the multidimensional walking aid was able to detect obstacles within the range of $0 \mathrm{~m}$ to $1 \mathrm{~m}$ at the left, right and front of the stick with an appropriate voice alert. 
In [10] the authors designed and implemented a microcontroller based mobility aid for visually impaired people. Their proposed stick consists of special detection sensors integrated to AT89C52 microcontroller for receiving, processing and sending signals to the alarm system. The system was designed, programmed with assembly language and tested for accuracy and checked by the visually impaired person. The hardware used consists of AT89C52 microcontroller, sensors (Ultrasonic, water, and light dependent resistor, LDR), and alarm. Their mobility aid designed for blind people is affordable, reliable and easy to operate. It reduces stress for people assisting the blind and provides comfort to blind during walking. The system consists of an ultrasonic sensor for obstacle detection, a water sensor for water detection in slippery areas and a light dependent resistor for dark detection. Each sensor is differentiated from one another through pattern of sounds. The cost effectiveness of the proposed solution leads to compromises in performance. The proposed method is a low cost and light weight system designed with a microcontroller that processes signal and alerts the visually impaired person over any obstacle, water or dark areas through beeping sounds. Their research work focuses on obstacle detection, light detection and water detection in order to reduce navigation difficulties for visually impaired people.

The study in [11] proposed the sensor assisted stick for the blind people. The main objective of this design is to develop an application for blind people to detect the objects in various directions, detecting pits and manholes on the ground to make free to walk. Their proposed method utilizes multiple sensors with features to detect the obstacle for collision avoidance, and to detect objects in all direction. Another sensor is placed near the bottom tip of the walking stick to detect pits on the ground. It integrated these sensors to the voice record and play chip. The prototype was modelled using Pro/E creo 5.0 Software. The hardware components used in the design are ATmega8 microcontroller, sensors, power supply unit, Servomotor, Buzzer, Voice record and play back device, and speaker. It is simple, cheap, configurable, and easy to handle intelligent guidance system. The results of the test on the system indicates that it is efficient in its capability to specify the source and distance of the obstacle. The prototype of the visually impaired aid is able to detect obstacles in all directions of the user. The proposed system has advanced features which detects front and top side of obstacles, pits and water stagnated/manholes on the ground. The implementation is quite expensive. It is not a cost-effective approach. Also, running this integrated set of hardware requires an alternative to the battery. The use of solar panels for instance, will be more advantageous in order to get recharged.

\section{Materials AND Methods}

This section deals with the theory of some of the components used as well as the design and implementation of an intelligent walking stick for the blind. The ultrasonic sensor transmitter generates signals and transmits them in a particular direction which will then be reflected back when they are approaching any obstacle(s), then the ultrasonic sensor receiver receives it and sends it to the microcontroller which will trigger/switch ON the Buzzer.

\section{Proposed System}

Our proposed system is made up of the Ultrasonic sensor was interfaced to the microcontroller, codes were written with the Arduino sketch and the physical sensor was connected to the microcontroller. The Arduino UNO is a microcontroller board based on the ATmega328p (datasheet). It has 14 digital outputs and inputs pins of which 6 can be used as PMW outputs, 6 analog inputs, a $16 \mathrm{MHz}$ quartz crystal, a USB connection, a power jack, an ICSP reset button. The Moisture sensor consists of two wire probes which rely on the specific resistance of water to sense its presence when there is a contact. The RF transmitter was interfaced with the microcontroller as codes were written with Arduino sketch and the RF receiver was connected to the microcontroller. The LCD was interfaced with the microcontroller connected to pin and all codes written with the Arduino sketch. The system will allow the blind to freely navigate to their desired destination. It is also user friendly and easy. It is affordable and therefore can be mass produced for use of the visually impaired. The system have the capacity to detect obstacles that exist on the ground during walks indoor and outdoor navigation. The smart stick, as shown in figure 2, is basically an embedded system integrating the following: pair of ultrasonic sensors to detect obstacles in front of the blind from ground level height to head level height in the range of $400 \mathrm{~cm}$ a head. Ultrasonic sensors and water sensors take real time data and send it to the microcontroller. After processing this data, the microcontroller activates the buzzer. The water sensor detect water on the ground, and battery is used to power the circuits.

The figure 3 below is the block diagram of the proposed system which shows the various components used in the design and implementation of the smart stick.

\section{Hardware}

The selection process of appropriate sensor depends on several factors such as, cost, atmospheric condition, kind of obstacle to be detected, detection range, and the desired precision of measurements collected information and its transmission frequency as shown in Tables 1 and 2. 


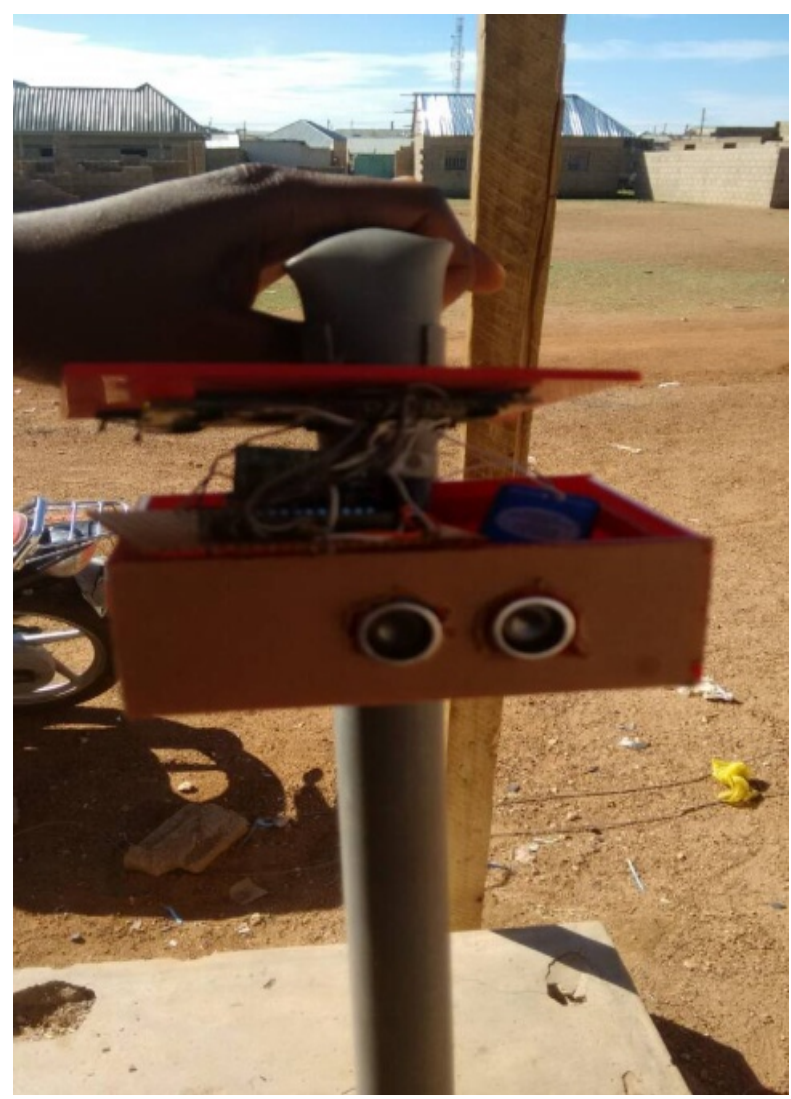

Fig. 2: A working ARDUINO smart blind stick

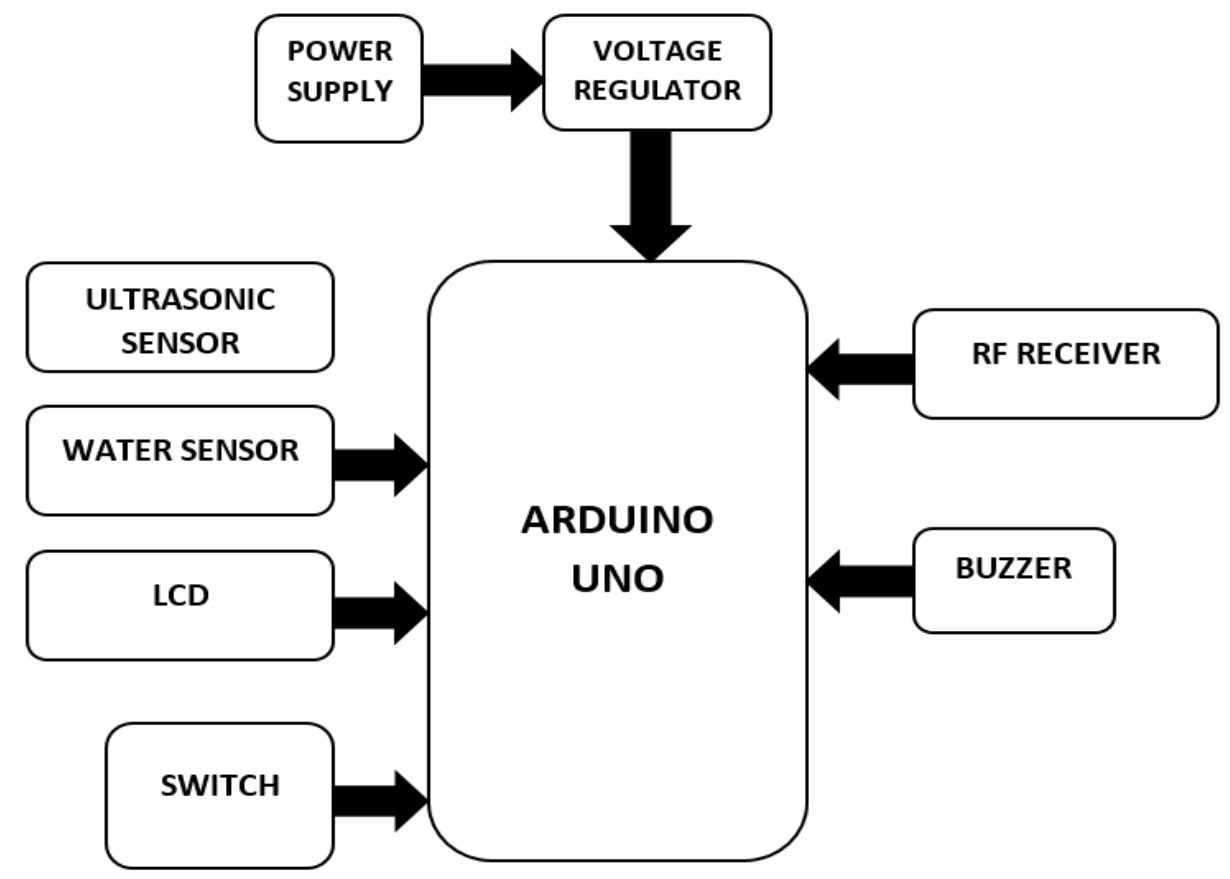

Fig. 3: A block diagram of the proposed system

The figures 4 and 5 show the block diagram of the RF transmitter block diagram and Circuit diagram of the system respectively. 


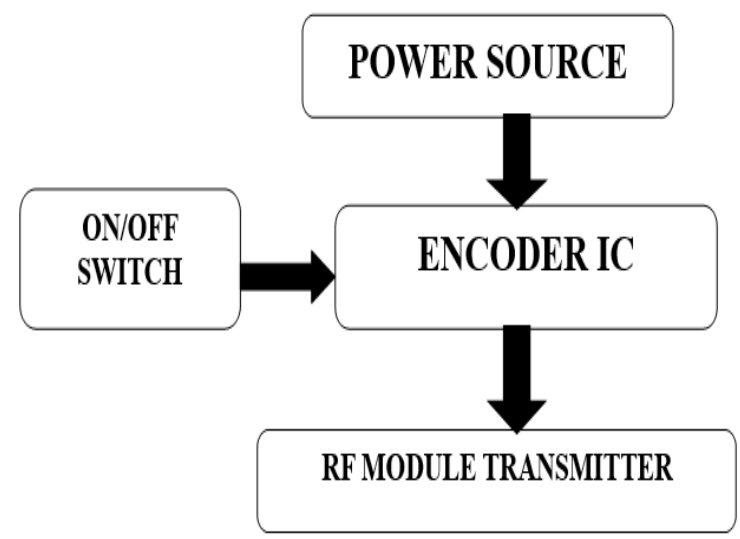

Fig. 4: The RF transmitter block diagram

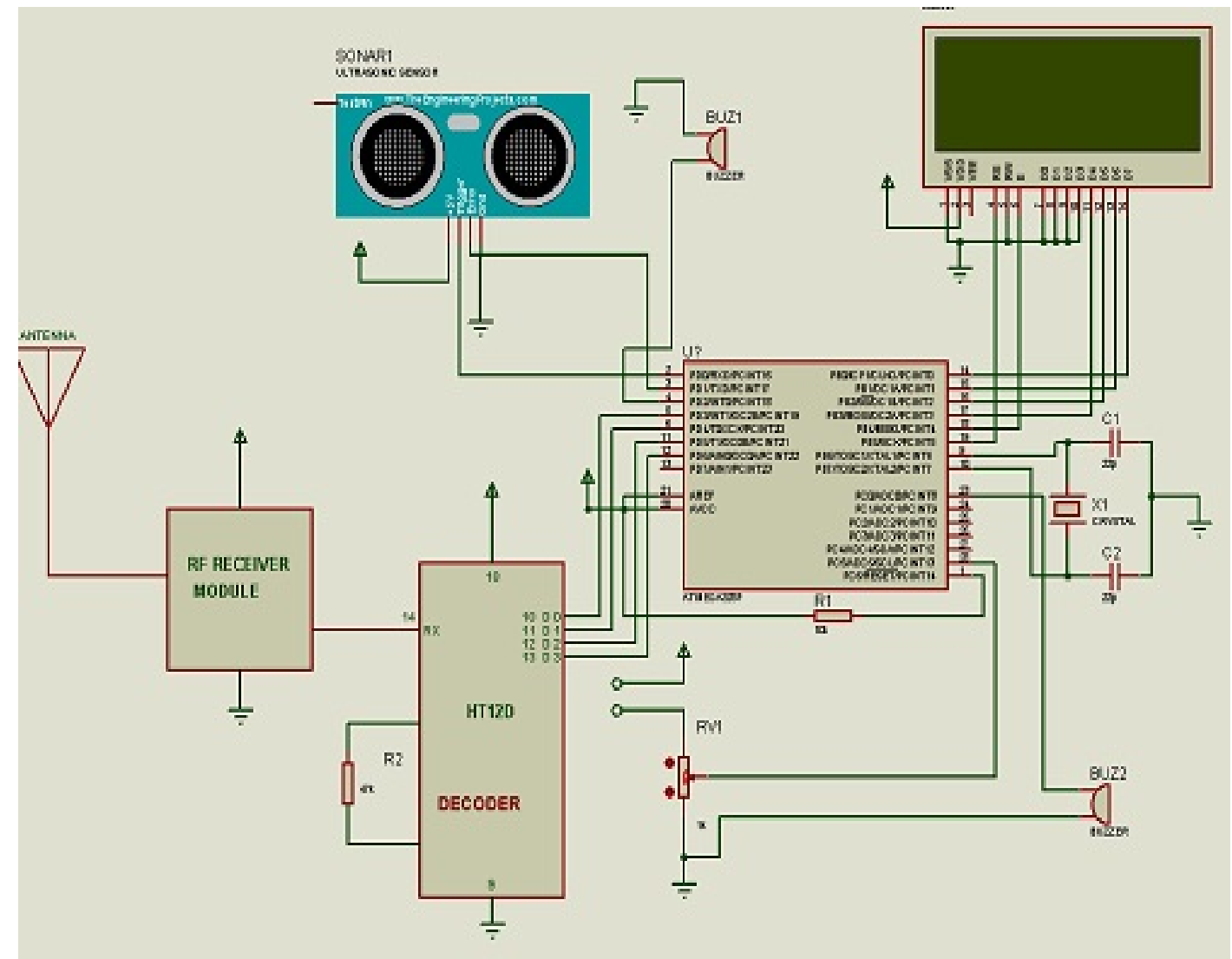

Fig. 5: Circuit diagram of the system 
The Circuit diagram of the RF transmitter is depicted in figure 6 below.

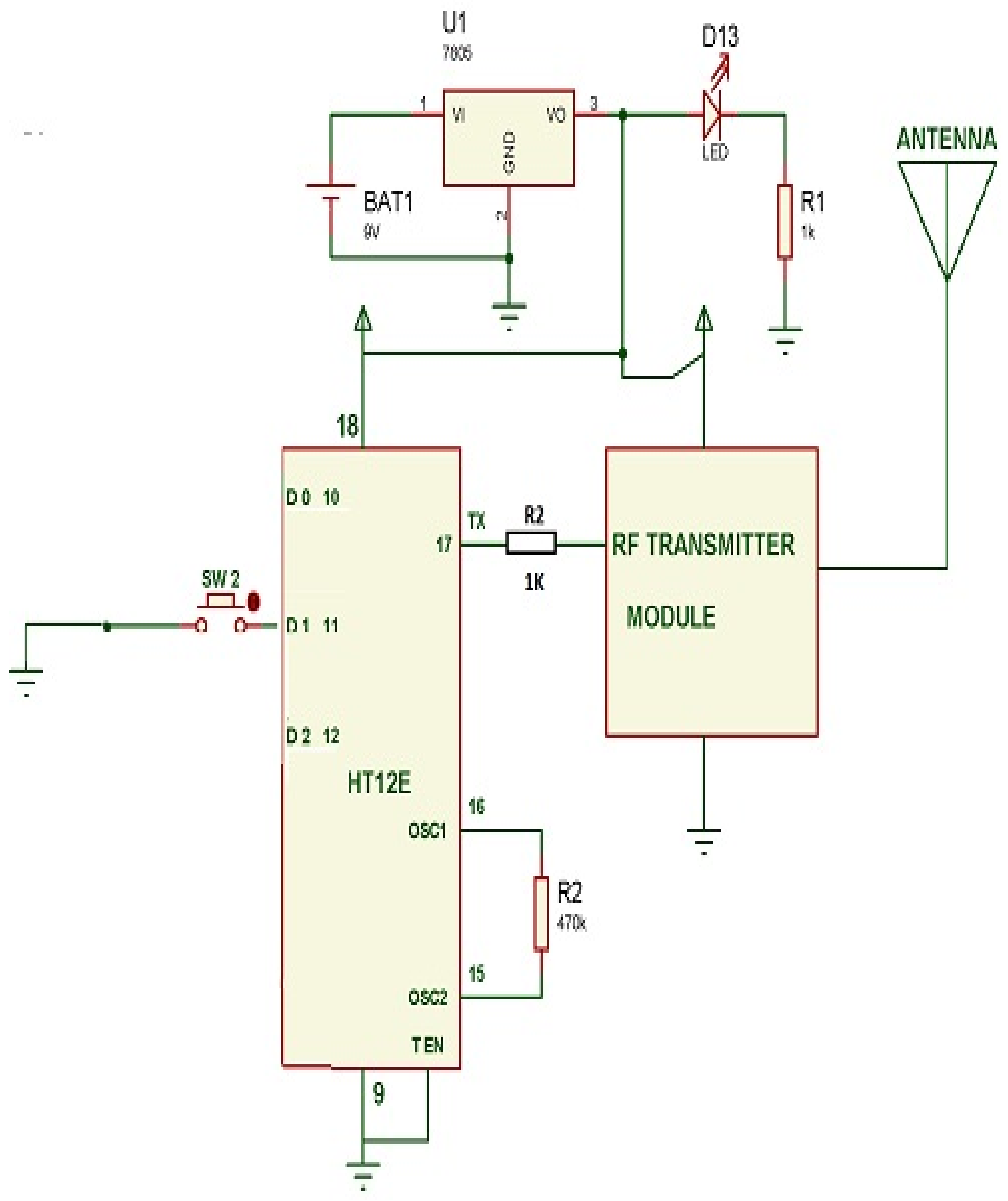

Fig. 6: Circuit diagram of the RF transmitter 
The flowchart of the proposed system is presented in figure 7 below.

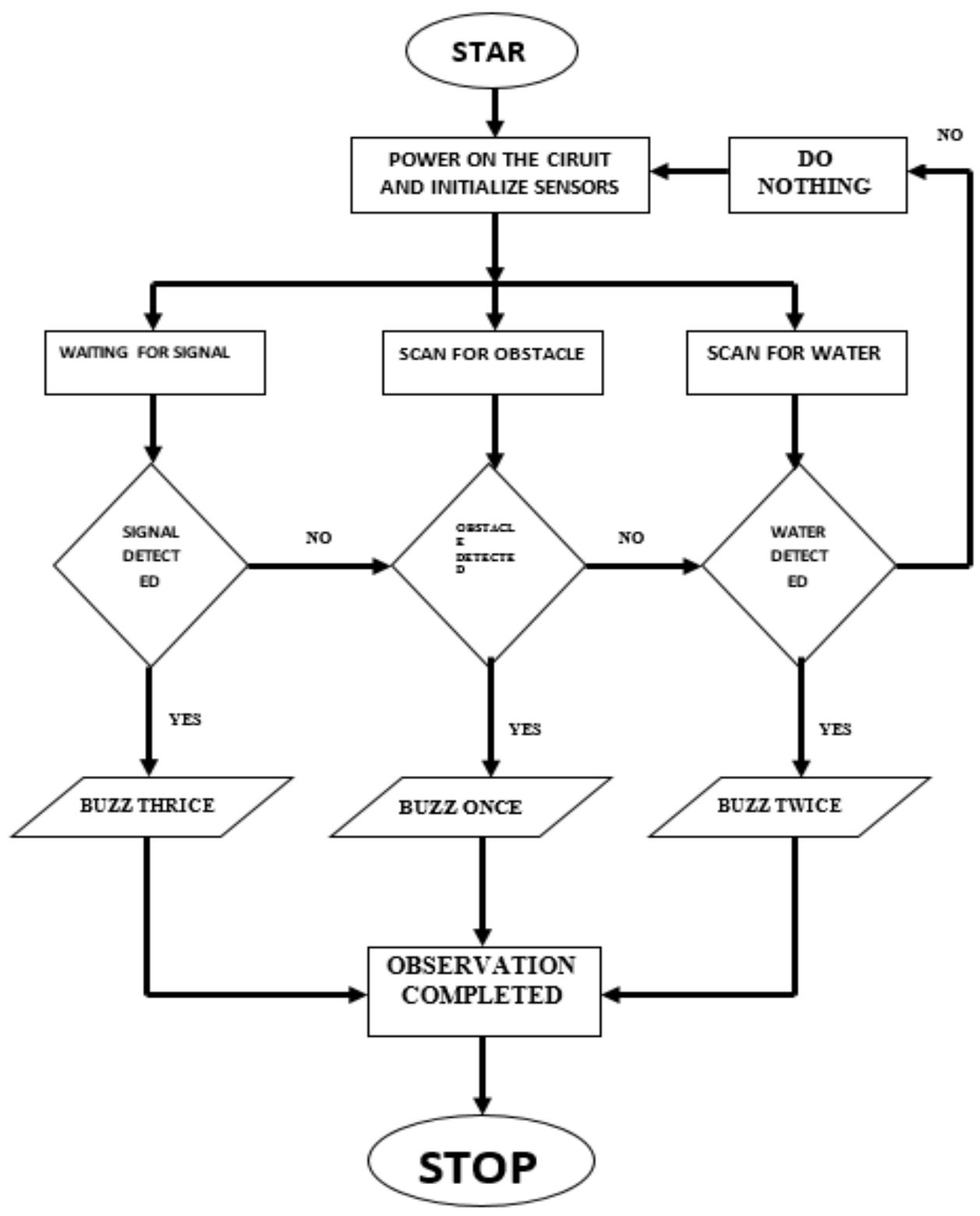

Fig. 7: Flowchart of the system

\section{III.RESULTS AND DISCUSSION}

The electrical circuit design was first assembled on a breadboard. After it was found intact on the breadboard, it was later transferred to a Vero board. The Vero board was divided into sections as such there is a power supply unit, display, transmitter, receiver and microcontroller section. Before soldering the components on the Vero board, the strip lines of the Vero board were cleaned with a razor blade to remove any grease, oxidation, oil and dirt. The resistors, capacitors, diodes as well as connecting terminals were carefully connected using soldering iron and soldering flux on the Vero board to avoid damage to integrated circuit IC sockets that were used. In soldering the components on the Vero board, care was taken to minimize component damage due to excessive heat from soldering iron. Also, adequate care was taken to avoid short-circuit between adjacent copper strips on the Vero board during soldering .In order to avoid possible short circuits, discontinuity of the copper strips was created when necessary, this is done by cutting the copper strips where necessary. 


\section{Testing of the Ultrasonic Sensor}

1. The circuit was connected on a bread-board.

2. An object was positioned (fixed at a distance) and the device was taken up to $1 \mathrm{~m}$ away from the object.

3. Gradually the distance between the device and object was varied until the distance at which the output voltage was logic high (i.e. Buzzer ON)

4. Step 3 and 4 were repeated for different objects of varying size.

\section{Simulation}

Several simulations were carried out to ascertain the efficiency of our proposed technique. The screenshot of the simulation of a working ultrasonic sensor is presented in figure 8 .

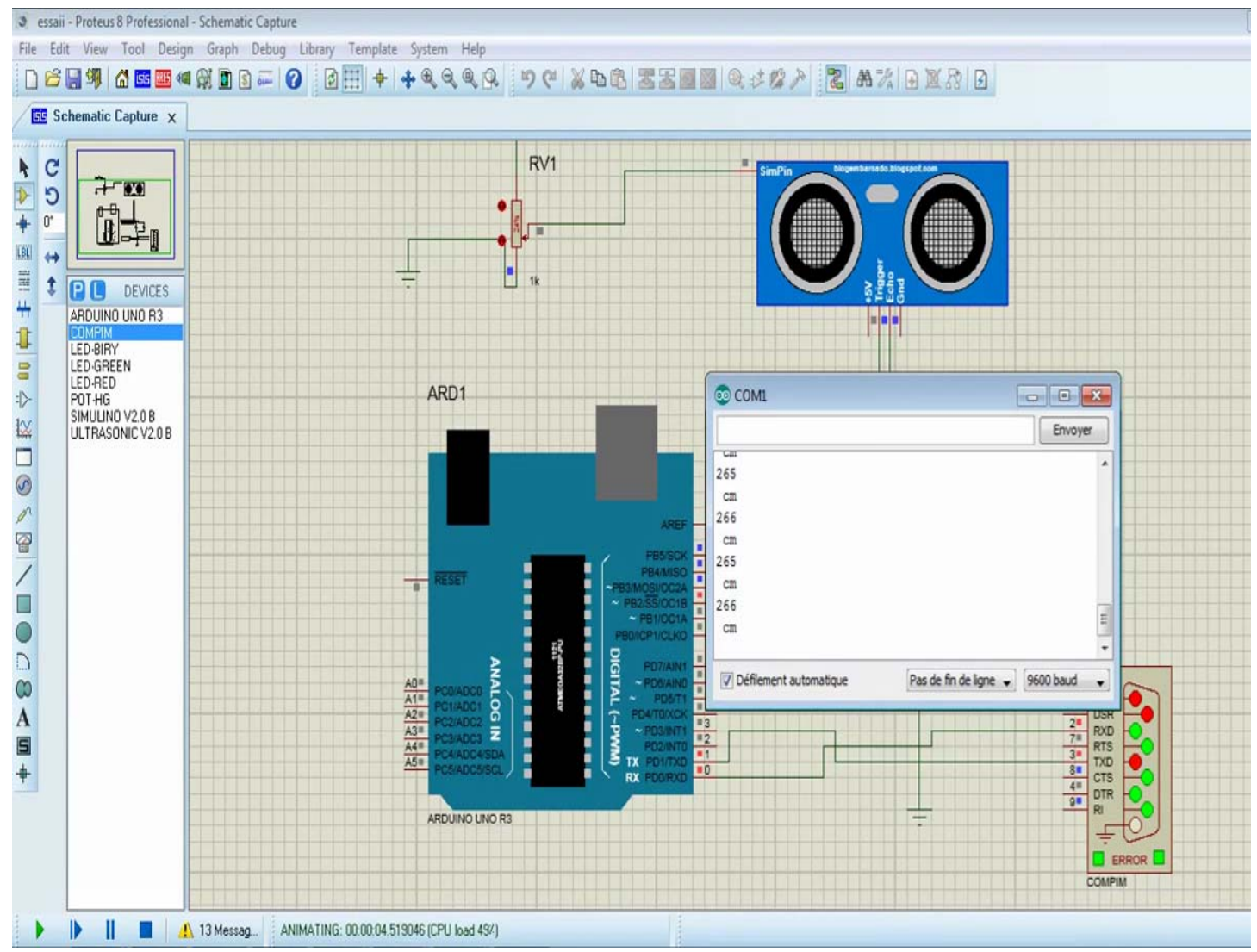

Fig 8: The simulation of a working ultrasonic sensor

\section{Testing of the Ultrasonic and Moisture Sensor}

Test and reliability check was conducted using each components of the smart stick to validate their efficiency and know if they are working according to specification before soldering.

1. Ultrasonic Sensor: The ultrasonic sensor was tested and the result as presented in Table 1 shows that the system worked according to specification at a distance not too far from the user. The buzzer came ON indicating the presence of obstacle on the way of the user.

2. Water Detection Sensor: A bread-board was used for connecting the circuit. A vessel containing water was placed on the table. The moisture sensor was immersed into the water contained and a beep sound was heard which is different from the beep from the obstacle detection. Table 2 depicts the result for water detection that prevent the user from entering into stationary water in the environment. Whenever the walking stick is inserted inside any deep water, the transistors will cause the alarm system to switch ON. The result of our test with moisture is depicted in figure 10. 
Table I. Ultrasonic Sensor Test Result

\begin{tabular}{|l|l|l|}
\hline S/No & Distance (Meters) & Alarm \\
\hline 1 & 0.5055 & ON \\
\hline 2 & 0.6036 & ON \\
\hline 3 & 1.1780 & ON \\
\hline 4 & 1.5732 & ON \\
\hline 5 & 1.8288 & ON \\
\hline 6 & 1.9700 & ON \\
\hline 7 & 2.0076 & ON \\
\hline 8 & 2.8850 & ON \\
\hline 9 & 3.0550 & OFF \\
\hline 10 & 3.7400 & OFF \\
\hline
\end{tabular}

Table 2. Water Detection Test Result

\begin{tabular}{|c|c|c|}
\hline Probes & No Water Detected & Water Detected \\
\hline Probe 1 & $\begin{array}{c}\text { 0v, the buzzer is inactive. } \\
\text { The transistor is off. }\end{array}$ & $\begin{array}{c}5 \mathrm{v} \text {, the buzzer came ON. } \\
\text { The transistor is forward bias. }\end{array}$ \\
\hline Probe 2 & $\begin{array}{c}\text { 0v, the buzzer is inactive. } \\
\text { The transistor is off. }\end{array}$ & $\begin{array}{r}5 \mathrm{v} \text {, the buzzer came ON. } \\
\text { The transistor is forward bias. }\end{array}$ \\
\hline
\end{tabular}

\section{Discussion of Results}

As ultrasonic sensors work on principle of echo, studying of its reflection on different obstacle is very important. The measurement cycle starts with microcontroller transmitting the $10 \mu$ s high level pulse to the sensor trigger pin to start ranging (T1), then the sensor will send out ultrasonic signal with $40 \mathrm{kHz}$ and $450 \mu \mathrm{s}$

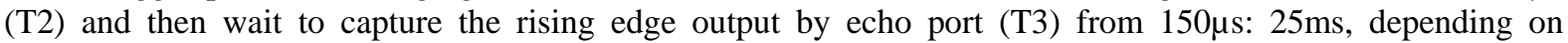
measured distance as shown in figure 9. In case of no obstacle (no signal reflected) it waits $38 \mathrm{~ms}$ before it restarts transmission. Ultrasonic distance sensor uses time of flight (TOF) to detect obstacle - the output is a digital pulse which length is the time it takes for the sound to reach the target and return before the beep is heard. The device was accurate with detecting obstacle of up to $2 \mathrm{~m}$. The water detector which is made up of two wires that which when came in contact with water would beep. They had to be touching the water before they are able to detect the water, which is why they were placed underneath the stick.
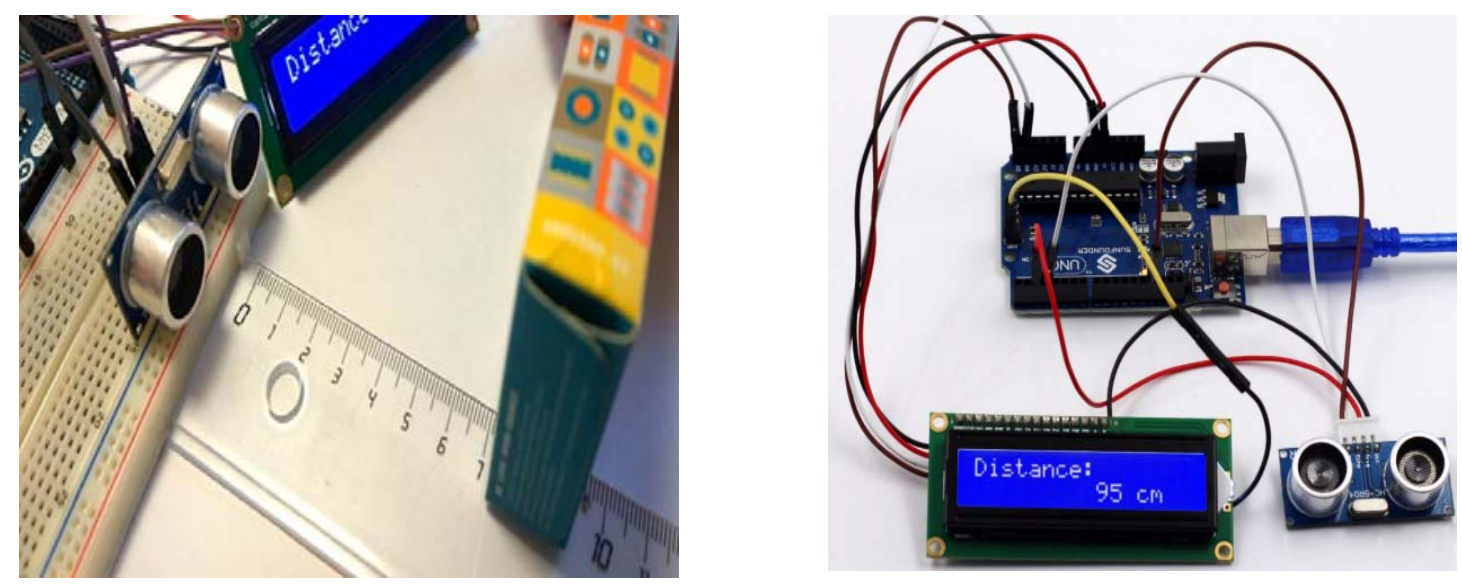

Fig. 9: Distance Measurement with the Circuit 


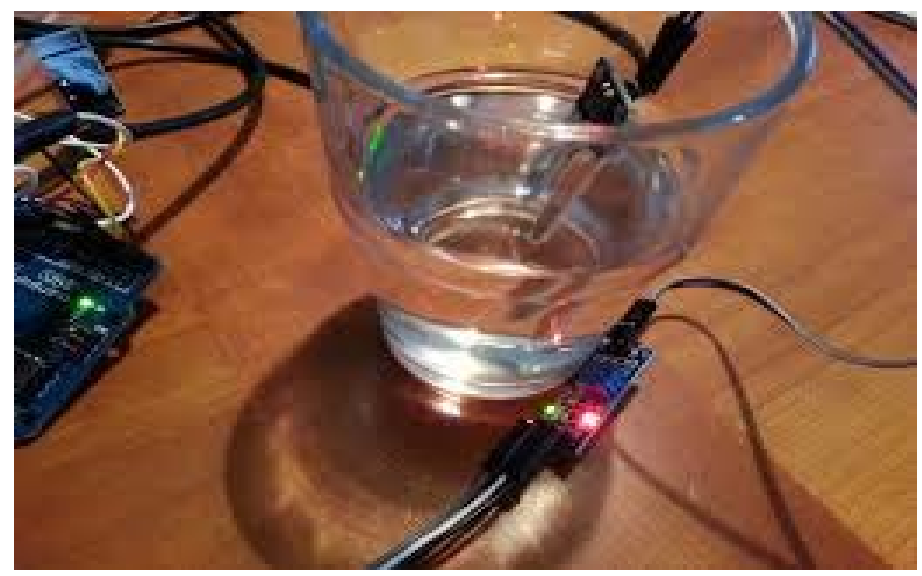

Fig. 10: Water Detection by the System

\section{IV.CONCLUSION}

It is worth mentioning at this point that the aim of this study which is the design and implementation of a smart walking stick for the blind has been fully achieved. The Smart Stick acts as a basic platform for the coming generation of more aiding devices to help the visually impaired to navigate safely both indoor and outdoor. It is effective and affordable. It leads to good results in detecting the obstacles on the path of the user in a range of three meters. This system offers a low-cost, reliable, portable, low power consumption and robust solution for navigation with obvious short response time. Though the system is hard-wired with sensors and other components, it's light in weight. Further aspects of this system can be improved via wireless connectivity between the system components, thus, increasing the range of the ultrasonic sensor and implementing a technology for determining the speed of approaching obstacles. While developing such an empowering solution, visually impaired and blind people in all developing countries were on top of our priorities. The device constructed in this work is only capable of detecting obstacles and moisture. Holes cannot be detected using this device nor the nature of obstacle. Therefore, a better device can be constructed using ultrasonic sensors, arduino Uno and other devices that employ audio commands to alert the user of what is in his path of movement. A vibrator may also be added for ease of use and convenience. In the future, further modifications to enhance the performance of the system will be added. These include: A global positioning method to find the position of' the user using the GPS, and GSM modules to communicate the location to a relative or care giver. It should also accommodate wide varying grips for flexible handling.

\section{REFERENCES}

[1] M. S. Nowak and J. Smigielski, "The Prevalence and Causes of Visual Impairment and Blindness among Older Adults in the City of Lodz, Poland.” Medicine, vol 94, number 5, pp. e505, February 2015 doi:10.1097/MD.0000000000000505

[2] G. Gayathri, M. Vishnupriya, R. Nandhini and M. Banupriya "Smart Walking Stick for Visually Impaired." International Journal of Engineering and Computer Science, vol. 3, number 3, pp. 4057-4061, 2014.

[3] R. Radhika, P.G. Pai, S. Rakshitha and R. Srinath "Implementation of Smart Stick for Obstacle Detection and Navigation." International Journal of Latest Research in Engineering and Technology, vol. 2, number 5, pp. 45-50, 2016.

[4] M.H. Mahmud, R. Saha and S. Islam "Smart Walking Stick - An Electronic Approach to Assist Visually Disabled Persons." International Journal of Scientific and Engineering Research, vol. 4, number 10, pp. 111-114, 2013.

[5] A. Jose, G. George, M.R. Nair, M. J. Shilpa and M. B. Mathai "Voice Enabled Smart Walking Stick for Visually Impaired." International Journal of Advanced Research in Electrical, Electronics and Instrumentation Engineering, vol. 5, pp. 80-85, 2016.

[6] R. Sheth, S. Rajandekar, S. Laddha and R. Chaudhari "Smart White Cane - An Elegant and Economic Walking Aid." American Journal of Engineering Research. Vol. 3, number 10, pp. 84-89, 2014.

[7] C.S. Kher, Y.A. Dabhade, S.K Kadam., S.D. Dhamdhere and A.V. Deshpande "An Intelligent Walking Stick for the Blind." International Journal of Engineering Research and General Science, vol. 3, number 1, pp. 1057-1062, 2015.

[8] B.G. Roopashree, B.S. Patil and B.R. Shruthi "Smart Electronic Stick for Visually Impaired.” International Journal of Innovative Research in Science, Engineering and Technology, vol. 4, number 7, pp. 6389-6395, 2015.

[9] O. O. Olakanmi, "A Multidimensional Walking Aid for Visually Impaired Using Ultrasonic Sensors Network with Voice Guidance", International Journal of Intelligent Systems and Applications (IJISA), vol. 6, number 8, pp. 53-59, 2014. DOI: 10.5815/ijisa.2014.08.06

[10] E. J. Chukwunazo and G. M. Onengiye "Design and Implementation of Microcontroller Based Mobility Aid for Visually Impaired People.” International Journal of Science and Research. Vol. 5, issue 6, pp. 680-686, 2015. Available at http://dx.doi.org/10.21275/v5i6.NOV164233.

[11] G. Prasanthi and P. Tejaswitha "Sensor Assisted Stick for the Blind People.” Transactions on Engineering and Sciences, vol. 3, number 1, pp. 12-16, 2015. 


\section{AUTHOR PROFILE}

Dada E. G. holds a PhD in Computer Science from the Department of Artificial Intelligence, Faculty of Computer Science and Information Technology at University of Malaya, Malaysia. He is a lecturer in the Department of Computer Engineering, University of Maiduguri, Nigeria. His current research interests include Soft Computing Algorithms (Particle Swarm Optimization, Fuzzy Logic, Neural Computing, Evolutionary Computation, Machine Learning, and Probabilistic Reasoning), Swarm Robotic, Optimization Techniques, Microcontroller and Information Security.

Shani A. I. is a graduate of the Department of Computer Engineering, University of Maiduguri, Nigeria. His research interests is computer architecture and microcontroller.

Adebimpe L. A. is a lecturer in the Department of Computer Science, Emmanuel Alayande College of Education, Oyo, Oyo State, Nigeria. He is currently a PhD. student in the Department of Computer Network and Security, University of Malaya, Malaysia. His research interests are in the areas of Steganography, Cryptography and Computer System. 